\title{
Téoros
}

Revue de recherche en tourisme

\section{Le rôle du Saint-Laurent dans le produit touristique québécois}

\section{Jean-Marie Lucas-Girardville}

Volume 6, numéro 2, juillet 1987

Le Saint-Laurent magnétique

URI : https://id.erudit.org/iderudit/1080506ar

DOI : https://doi.org/10.7202/1080506ar

Aller au sommaire du numéro

Éditeur(s)

Université du Québec à Montréal

ISSN

0712-8657 (imprimé)

1923-2705 (numérique)

Découvrir la revue

Citer cet article

Lucas-Girardville, J.-M. (1987). Le rôle du Saint-Laurent dans le produit

touristique québécois. Téoros, 6(2), 15-17. https://doi.org/10.7202/1080506ar d'utilisation que vous pouvez consulter en ligne.

https://apropos.erudit.org/fr/usagers/politique-dutilisation/ 


\section{Le rôle du Saint-Laurent dans le produit touristique québécois}

\section{par Jean-Marie Lucas-Girardville *}

Le Saint-Laurent, c'est mille kilomètres de rivages, cent kilomètres de large à l'embouchure, des milliards de mètres-cubes d'eau - et de bien d'autres choses - occupant plus de $155000 \mathrm{~km}^{2}$. C'est $9 \%$ du territoire québécois et cinq fois la superficie de la Belgique.

Nous voilà donc en face d'un monstre.

Mais son importance n'est pas seulement physique, elle est aussi économique, sociale, culturelle et climatique.

Car le Saint-Laurent, $c^{\dagger}$ est aussi un demimillénaire de notre histoire, le site privilégié de nos entreprises industrielles et de nos. grandes villes, le rendez-vous des oies sauvages, des baleines et des saumons, le terrain de jeu des Québécois et l'antichambre des Grands Lacs.

Par son omniprésence, par ses multiples visages et ses fonctions diversifiées, on ne peut ni l'ignorer, ni l'éviter dès lors que l'on parle de tourisme et de voyages. Découvrir le Québec sans avoir découvert le Saint-Laurent, ce serait passer à côté de son vécu, de sa culture, de ses paysages, et bien souvent de ses habitants. Ce serait en quelque sorte... manquer le bateau!

Mais en pratique, comment une entite aussi vaste et complexe peut-elle $\mathrm{s}^{*}$ intégrer à notre produit touristique? À quels niveaux du developpement touristique le SaintLaurent entre-t-il en jeu?

\section{"Des"' Saint-Laurent}

Il faut considérer le Saint-Laurent, non pas comme une seule entité homogène, mais comme plusieurs éléments distincts ayant leur propre personnalité et reliés par un méme fil conducteur: l'eau.

Ainsi, il $n^{\prime} y$ a pas un Saint-Laurent, mais "des" Saint-Laurent: tantôt rivière, tantôt rapides, tantót lac, tantôt canal, tantôt fleuve et tantôt mer, il peut être langoureux ou déchaîné selon le lieu et l'heure. On ne peut vraiment pas dire précisément où il "Jean-Marie Lucas-Girardville, Coordonnateur, Dáve-
loppement regional, Direction de l'analyse et du developpement, ministaré du Tourisme du Qubboc. commence et où il finit: la marée se fait sentir jusqu'à Trois-Rivières mais l'eau devient salée en aval de Québec, et son embouchure est quelque part entre Tadoussac et Forillon, là où il devient aussi large que la péninsule gaspésienne.

Où finit le fleuve et où commence la mer? Pour les riverains, entre l'île aux Coudres et Trois-Pistoles; pour les touristes, entre Trois-Pistoles et Matane: tout dépend des odeurs, des vagues et de l'éloignement des rives.

Le Saint-Laurent a donc plusieurs natures et plusieurs visages qui ont forgé autant de races d'hommes, de femmes et de types $\mathrm{d}^{\dagger}$ établissements humains.

Et chaque type de touristes peut y trouver son compte: le touriste urbain aimera fläner dans les Vieux-Ports de Québec et Montréal, le touriste de plein air sera comblé par le cap Tourmente ou les illes de Sorel. Le touriste de circuits s'émerveillera devant les paysages de Charlevoix ou les villages du Bas-Saint-Laurent, tandis que l'amateur de stations touristiques pourta passer quelques jours a Tadoussac. Les chasseurs d'oiseaux migrateurs trouveront leur gibier sur les battures du lac SaintPierre et les pêcheurs taquineront la morue aux Escoumins.

À chacun son Saint-Laurent, et à chacun son médium: l'automobile au bord de l'eau pour les itinérants; la randonnée pédestre sur les plages ou les rochers; les croisièresexcursions pour admirer les villes de Québec ou de Montréal aussi bien que les oies sauvages ou les baleines; les traversiers pour relier les deux rives; la bicyclette sur les routes riveraines moins accidentees ou sur les îles; les bategux de plaisance, la planche à voile ou la plongée sous-marine pour les actifs; le seau et la pelle pour les petits...

Bref, le Saint-Laurent se prête à divers styles de tourisme (séjour, circuit) pour différents types de touristes, (actifs, contemplatifs) en offrant des éléments attractifs en tous genres (naturels, culturels, urbains, récréatifs) et même des éléments de support (transport des marchandises et des personnes).
Un potentiel ou une contrainte Il $y$ a différentes façons d'évaluer le SaintLaurent.

Comme tout symbole, on peut le glorifier de qualificatifs lyriques et ronflants, ou le décrire de façon purement réaliste et bassement terre à terre, selon qu'on est poète ou matérialiste, aventureux ou prudent, riverain ou touriste.

Certains diront: " Le Saint-Laurent, c'est beau, c'est majestueux, c'est grandiose, c'est riche de decouvertes, c'est unique, c"est la colonne vertébrale du Québec" . On pourrait leur rétorquer que "Le SaintLaurent, c'est un grand fleuve parmi bien d'autres: le Nil, l'Amazone, le Mississipi, le Yang-Tsé, le Rhin, aussi riches et aussi beaux. C'est trop long et trop loin, c'est triste, $c^{+} e s t$ froid, c'est dangereux et $c^{4} e s t$ sale car c'est le tube digestif du Québec'. Pour l'industrie touristique québécoise estce vraiment un atout?

Un touriste étranger planifiant ses prochaines vacances pourrait poser quelques questions embarrassantes: "tPeut-on se baigner dans le Saint-Laurent? Quelle est la temperature de l'eau? Combien de temps fautil pour aller des Grands Lacs à Québec en voilier? Est-ce prudent de naviguer en aval de l'île d'Orléans? Combien de plaisanciers ont été secourus par la garde-côtièré ces dernières années? Quelle distance en auto faut-il parcourir pour aller jusqu'aux îles de Mingan? Peut-on revenir par la rive sud ou faut-il refaire le méme chemin? Est-ce qu'il y a beaucoup d'établissements au bord de l'eau? $\mathrm{Y}$ a-t-il des pistes cyclables longeant le fleuve ou la mer? Des sentiers de randonnées? Quelles plages de sable sont aménagées? Peut-on visiter les iles du Saint-Laurent? Les entreprises fonctionnent-elles en septembre-octobre? Quel temps fait-il à cette époque?".

Répondre à toutes ces questions, ce serait énumérer une foule de contraintes et d'obstacles qui tendent à faire du Saint-Laurent un beau potentiel laissé pour compte à cause de difficultés plus ou moins grandes, voire de négligences.

Il faut dire que ces contraintes sont en 
grande partie physiques, et done incontrólables: éloignement, climat, brièveté de la saison, courants glaces et violents, hauts fonds, marées, etc. D'autres sont inhérentes à la gestion du territoire: droits seigneuriaux sur les berges, privatisation de certaines îles (ìle aux Oies, aux Lièvres) ou statut particulier (Grosse Ile, Anticosti), absence de traversier (Isle Verte, Anticosti), de quais ou de ports de refuge.

$D^{\prime}$ autres inconvénients relèvent plus d'une absence de moyens ou de volonté; allors que la cóte américaine regorge d'hôtels et de "tourist houses" " sur la plage, on construit trop souvent les établissements d'hébergement ou de restauration loin du bord de l'eau, et souvent méme sans vue sur le fleuve ou sur la mer. Combien d'hôtels ou d'auberges de Charlevoix sont au bord de $1^{\dagger}$ eau? On ne peut même pas descendre directement du Manoir Richelieu sur la rive, trente métres plus bas! En Gaspésie, il est souvent difficile de se loger ou se nourrir sans avoir la route au premier plan.

Tant que le Saint-Laurent ne sera pas mieux choyé, tant qu"on ne le "sacralisera" " plus et mieux, tant que les intervenants touristiques ne considéreront pas son acciss et son contact comme un privilege convoite par les touristes, il ne pourra devenir un produit touristique.

\section{Le rôle du Saint-Laurent dans le produit touristique}

Le produit touristique est composé d'élements attractifs et d'éléments de support. Le Saint-Laurent est, certes, un flément attractif de première importance mais, quel que soit son degré d'intérêt tconomique, góographique, culturel ou récréatif, il reste une simple ressource. Une ressource diversifiée comme peuvent l'être la forêt qué becoise ou le massif laurentien, et qui ne deviendra produit que lorsqu'elle sera utilisée, exploitée, aménagée, transformée et surtout vendue comme un tout sur les marchés touristiques, car un produit est une marchandise commercialisable de façon autonome.

Néanmoins, le Saint-Laurent joue d'ores et déjà un rôle important dans le produit touristique québécois et, ce, de plusieurs façons.

Tout d'abord comme élément attractif aux multiples facettes, ainsi qu'on l'a déjà dit. Mais au-dela de cette considération, le Saint-Laurent joue d'autres rôles, tantôt positifs, tantôt négatifs:

- c'est un elément unificateur entre les treize régions touristiques qui le bornent, dans l'axe est-ouest. C'est en quelque sorte leur fil conducteur, la brochette sur laquelle les régions se positionnent pour être "consommees" l'une apres l'autre;

- c'est une pierre d'assise, seul point entre les produits touristiques des régions sud et ceux de leurs vis-ă-vis respectifs de la rive nord;

- c'est un élément diviseur: il constitue une barrière physique importante entre le Québec du Nord et celui du Sud, relies par des ponts en trois endroits seulement sur 1,000 kilometres. Ailleurs, les traversiers constituent une contrainte que les touristes n'ont pas tous le cou- rage d'affronter (prix, durée, attente, mal de mer parfois);

- c'est aussi un élément diviseur intrarégional parce que, dans chaque région touristique riveraine, le produit oótier se distingue de celui du haut-pays sur bien des points et qu'en outre les flux touristiques sont très réticents à s'éloigner de la rive pour s'enfoncer dans des terres peu connues et peu exploitées.

Cette disparité intrarégionale, on peut la sentir dans toutes les régions, car l'intérieur des terres offre des produits spécifiques orientés sur le séjour-destination, alors que la côte offre un tourisme polyvalent de circuit et de passage. En ce sens, le SaintLaurent a tendance à voler la vedette à l'arrière-pays. On peut toutefois considérer cette dichotomic comme une complementarité et, par conséquent, comme un atout pour chaque région: la présence du Saint-Laurent attire des clientèles que les zones de l'intérieur n'ont qu'à drainer et retenir. Dans cette perspective, les plans de développement touristique régionaux (ainsi que les schémas d'amenagement des municipalités régionales de comté) devraient permettre de mieux intégrer le développement et le marketing des produits de la côte et de 1 'intérieur.

Quoi qu'il en soit, le Saint-Laurent se voit cantonne actuellement à un rôle de ressource brute et d'outil de positionnement. Comme élément physique unificateur de treize des dix-huit régions touristiques du Québec, il pourrait étre avantageusement utilisé comme image de marque auprès des marchés etrangers: il deviendrait ainsi un

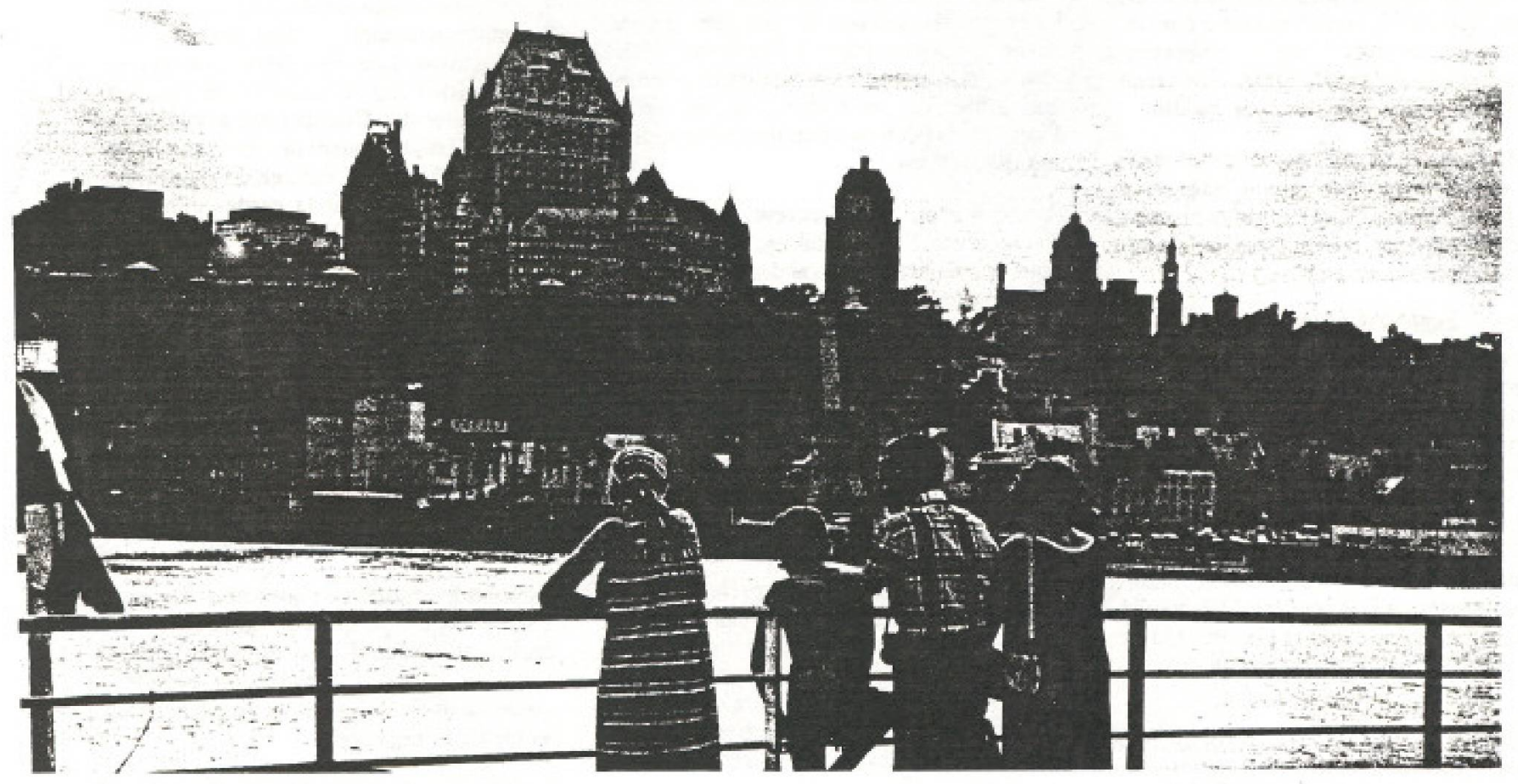

Trwersec entre Lovis et Gudbac. 
caractère distinctif majeur du Québec, bien qu'il posséde peu d'avantages comparatifs.

Lieu de convergence des deux-tiers de la population du Québec et lieu de partage des terres, il rassemble tout ce que nous avons de relief, de flore, de faune et de caractères humains, des quartiers cosmopolites de Montréal aux réserves indiennes en passant par les villages de la Nouvelle-France. Lieu privilégié des migrations, celles des humains, des navires, celles des baleines, des saumons, des oies blanches et celles des glaces, il devient le lieu de prédilection de ces migrants que sont les touristes.

Mais c'est à la façon dont on l'amenagera et dont on l'exploitera que cette ressource deviendra ou non un produit touristique, ou plutôt des produits touristiques s'adressant à des segments de marchés spécifiques. Car, plus la ressource est multiple, plus les produits sont nombreux et les clientẻles diversifiées. Il faudra donc respecter les particularismes de chacune d'elles.

Si l'on examine par exemple le marché américain du tourisme d'agrément, on constate que le segment des "circuits touristiques" présente le plus fort potentiel pour le Québec en termes de nuitées ${ }^{(1)}$. C'est une considération très importante dans les perspectives de développement et d'aménagement du Saint-Laurent.

À court terme, ce qu'il faut privilégier c'est le contact avec le fleuve: contact visuel, contact auditif et olfactif. Dans un second temps, c'est la création d'une ambiance: atmosphère de souvenirs et de mystère, sentiment de force de la nature.

Pour les marchés touristiques, qu'ils soient du Québec ou d'ailleurs, il faut développer avant tout des croisières-excursions qui permettent de voir ce qui est invisible de la côte (les baleines, les anses, les îles, les caps) et de sentir le Saint-Laurent de l'intérieur.

II faut aussi prioriser tout ce qui favorise le contact immédiat avec l'eau, sans barrière: sentiers de randonnée pédestre sur les rives, pistes cyclables, établissements d'hébergement et de restauration au bord de l'eau, avec terrasses et verrières, pêche sur les quais. Il faut inciter les touristes de circuits à sortir des grands axes et à emprunter les routes panoramiques (la 132, le Chemin du Roy), a bifurquer vers les fenêtres ouvertes sur le fleuve (Cap-auxOies, Saint-Joseph-de-la-Rive, PetiteRivière-Saint-François,Saint-Fabien-surMer,Pointe-aux-Outardes, Pointe-à-laRenommée, etc.) et à se rendre sur les îles. Le Saint-Laurent doit cesser d'être une barrière et pour qu'on le traverse de plus en plus, il faut créer des circuits interregionaux complémentaires. Le circuit de la Côte-Nord, par exemple, deviendra intéressant lorsqu'il sera possible de revenir par la Gaspésie après avoir visité Anticosti et le Parc National des Iles de Mingan (ou inversement). Dans un autre ordre d'idées, les régions de Charlevoix, du Bas-SaintLaurent et du Pays-de-1'Érable pourraient s'associer au traversier de SaintSiméon/Rivière-du-Loup pour créer un circuit homogène.

Montréal et Québec devront continuer d'orienter leurs interventions de façon à offrir aux touristes urbains des aménagements attrayants qui leur donneront le goût de flâner sur des quais agrémentés d'éléments d'animation et intégrés physiquement et touristiquement au milieu urbain environnant (habitations, commerces, transports en commun, activités culturelles,

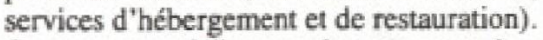
Quant aux croisières au long cours, c'est plus par des croisières intégrant l'hébergement existant le long du Saint-Laurent qu'on exercera un impact économique sur le Québec qu'avec des navires tout équipés qui tiennent leurs clients captifs. Un système de croisage-cabotage avec des navires de taille moyenne bien équipess et débarquant les passagers à chaque nuit aurait pour effet de rentabiliser les établissements riverains comme on le fait avec les autobus nolisés par des grossistes.

Le développement du réseau de navigation de plaisance ne devrait pas être une priorité touristique sur le Saint-Laurent, compte tenu de divers facteurs déja exposés dans Le tourisme au Québec ${ }^{(2)}$. Seul le tronçon Hull-Montréal présente un potentiel à court terme, compte tenu de sa proximité, mais il n'est pas sur le Saint-Laurent. Quant au tronçon du Saint-Laurent entre Montréal et le lac Ontario, il est en Ontario et sert principalement aux plaisanciers québécois à s'exiler vers le sud, en dépit des aménagements nautiques existants jusqu'à Québec.

Malheureusement, au Québec, le potentiel nautique touristique le plus intéressant est situé dans la zone du Saint-Laurent où les contraintes d'éloignement, de température et de navigation sont les plus fortes, c'està-dire en aval de Québec, et là où les plaisanciers locaux sont les moins nombreux pour justifier les équipements.

C'est plus par des aménagements légers, ponctuels, mais bien ciblés que le SaintLaurent conquerra ses titres de noblesse touristique. Il doit apprivoiser les touristes en les attirant tout contre lui, par des mesures incitatives, une sacralisation des sites et un accès facile plus que par des investissements lourds. Par exemple, les guides touristiques régionaux produits par le ministère et les ATR ne mentionnent mème pas si un établissement d'hébergement est au bord de l'eau!

Et que dire de la qualité visuelle! En ce domaine, chacun a sa responsabilité. Si l'on prétend faire du Saint-Laurent un produit touristique, il faut alors, comme pour tous les produits, qu'il soit attrayant et que son emballage soit soigné. Ce sont les riverains, les investisseurs et les municipalités qui ont une responsabilité à assumer au niveau de l'aménagement, de la propreté, de l'affichage, des normes de construction (hauteur, formes, couleurs) et de l'accès public aux rives.

On n'insistera jamais assez sur l'importance de la pollution visuelle. Il suffit de longer le Saint-Laurent de Montréal à Forillon pour se désoler des gâchis accomplis par inconscience, par ignorance ou par indifférence. La compagnie pétrolière Irving. par exemple, est un modele dans le genre.

Des menaces pèsent aussi sur certains de nos attraits: les baleines, les poulamons, les saumons peuvent étre affectés par la température de l'eau et la pollution résultant des barrages ou des industries.

Mais l'un des problèmes majeurs auxquels fait face le développement touristique du Saint-Laurent, c'est l'incroyable diversité des intervenants concernés: diversité géographique (ATR, MRC, municipalités) et administrative (ministères du Tourisme, du Loisir, de la Chasse et de la Pêche, de l'Environnement, Pêcheries et Agriculture, Pêches et Octans Canada, Parcs Canada, Transports Québec et Canada, entreprises, riverains, Garde côtière, Douanes et accises).

Faire du Saint-Laurent un produit touristique, cela semble parfois une utopie, un idéal impossible à atteindre, tant il y a d'obstacles. La seule vraie façon d'y parvenir, ce serait bel et bien de boucher le détroit de Belle-Isle entre.Terre-Neuve et le Labrador, et faire ainsi grimper la température moyenne du Québec de $4^{\circ} \mathrm{C}$ !

Car il ne faut pas rêver en couleurs: dans la plupart des cas, il y a des contraintes non influençables. Pour investir dans une ressource de façon à la transformer en produit touristique, il faut une perspective de rentabilité. Or, dans bien des coins du SaintLaurent, les contraintes physiques et la faiblesse du bassin de population locale ne permettent pas d'envisager un retour suf-

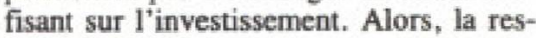
source restera à l'état brut, indomptée, inapprivoisée.

Après tout, est-ce que ce ne sera pas mieux ainsi? Et n'est-ce pas là, au fond, la vraie vocation du Saint-Laurent?

Un monstre, ça ne se laisse pas apprivoiser comme ça! $f$

\section{Rofbrancas}

(1) Canada, Expansion industriela et rofgionale et Québec, ministère du Tourisme, Le marchb ambricain des voyages d'agrtment, Québec, $1987,250 \mathrm{p}$.

(2) Ministère du Tourtarne du Cuebec, Le tourkme au Qubbeci blien ot perapectives d'action. 1985-1988, Outbec, 1984, 94 p. 